\title{
Plants as a Source of Therapeutic and Health Products
}

\author{
Philippe Christen and Muriel Cuendet ${ }^{\star}$
}

\begin{abstract}
The research unit of Pharmacognosy is mostly working on the biological activity and safety of natural products, as well as herbal dietary supplements. One of the focuses relies on finding new cancer chemopreventive compounds by means of a battery of short-term in vitro bioassays developed to monitor inhibition of tumorigenesis at various stages. Neglected diseases are a major problem in developing countries. Therefore, the search for new or improved treatments is also needed and consists of another area of research of the unit. For both projects, in-depth mechanistic studies, as well as in vitro and in vivo absorption and metabolization experiments are performed. Problems related to undeclared, unauthorized or toxic botanicals in herbal dietary supplements are of growing importance as they generally have not gone through a rigorous drug testing process as for therapeutic phytochemicals. For this, a generic method was developed for the multi-targeted screening of biomarkers which aims at characterizing plant species in these supplements.
\end{abstract}

Keywords: Cancer chemoprevention · Dietary supplements $\cdot$ Natural products $\cdot$ Neglected diseases

\section{Cancer Chemopreventive Compounds from Natural Products}

It is generally recognized that the lifetime probability for an individual to develop some form of cancer is in the range of 50 to $70 \%$. When etiological factors are well established, such as overexposure to ultraviolet irradiation or cigarette smoking, primary methods of prevention can be used. Conversely, when the etiology remains unknown, such as for breast and prostate cancer, alternate methods of prevention are highly desirable. Cancer is considered the end stage of a chronic disease process characterized by abnormal cell and tissue differentiation. ${ }^{[1]}$ This process of carcinogenesis eventually leads to the final outcome of invasive and metastatic cancer. Recent advances in defining cellular and molecular levels of carcinogenesis have led to the development of cancer chemoprevention. ${ }^{[2,3]}$ Cancer chemoprevention is defined as the use of synthetic or natural agents to inhibit, retard, or reverse carcinogenesis, or prevent the development of invasive cancer. ${ }^{[4]}$ Rational and successful implementation of chemopreventive strategies relies intrinsically on tests for efficacy and mechanistic assays, as well as availability of promising chemopreventive agents, reliable intermediate biomarkers,

${ }^{\star}$ Correspondence: Prof. M. Cuendet

School of Pharmaceutical Sciences

University of Geneva, University of Lausanne

30 Quai Ernest-Ansermet

$\mathrm{CH}-1211$ Geneva

Tel.: +41223793386

Fax: +41223793399

E-mail: muriel.cuendet@unige.ch and appropriate clinical cohorts to discover safe and effective drugs for primary and secondary prevention of human cancers. ${ }^{[5]}$ Dietary consumption of foods and herbal medicines is a convenient method of administrating potentially beneficial phytochemicals in a cost-effective manner.

Plants and other materials from nature are evaluated for their potential chemopreventive activity using a battery of short-term in vitro bioassays developed to monitor inhibition of tumorigenesis at various stages. For the initiation stage, induction of $\mathrm{NAD}(\mathrm{P}) \mathrm{H}$ :quinone reductase (QR) activity ${ }^{[6]}$ is assayed. For the promotion stage, inhibition of activities such as

$\mathrm{NF}-\kappa \mathrm{B}$ and nitric oxide (NO) production ${ }^{[7]}$ are determined. For the progression stage, inhibition of histone deacetylase (HDAC) activity ${ }^{[8]}$ is measured. In collaboration with J.-L. Wolfender (EPGL, University of Geneva), active samples are then microfractionated to identify novel and otherwise unpredictable chemical entities with cancer chemoprevention activities. ${ }^{[9]}$ Once an interesting compound has been identified, a targeted isolation using a large amount of plant material is performed in order to get a sufficient amount of pure compound to do in-depth mechanistic studies. In vitro and in vivo absorption and metabolization experiments are also performed (Fig.1).

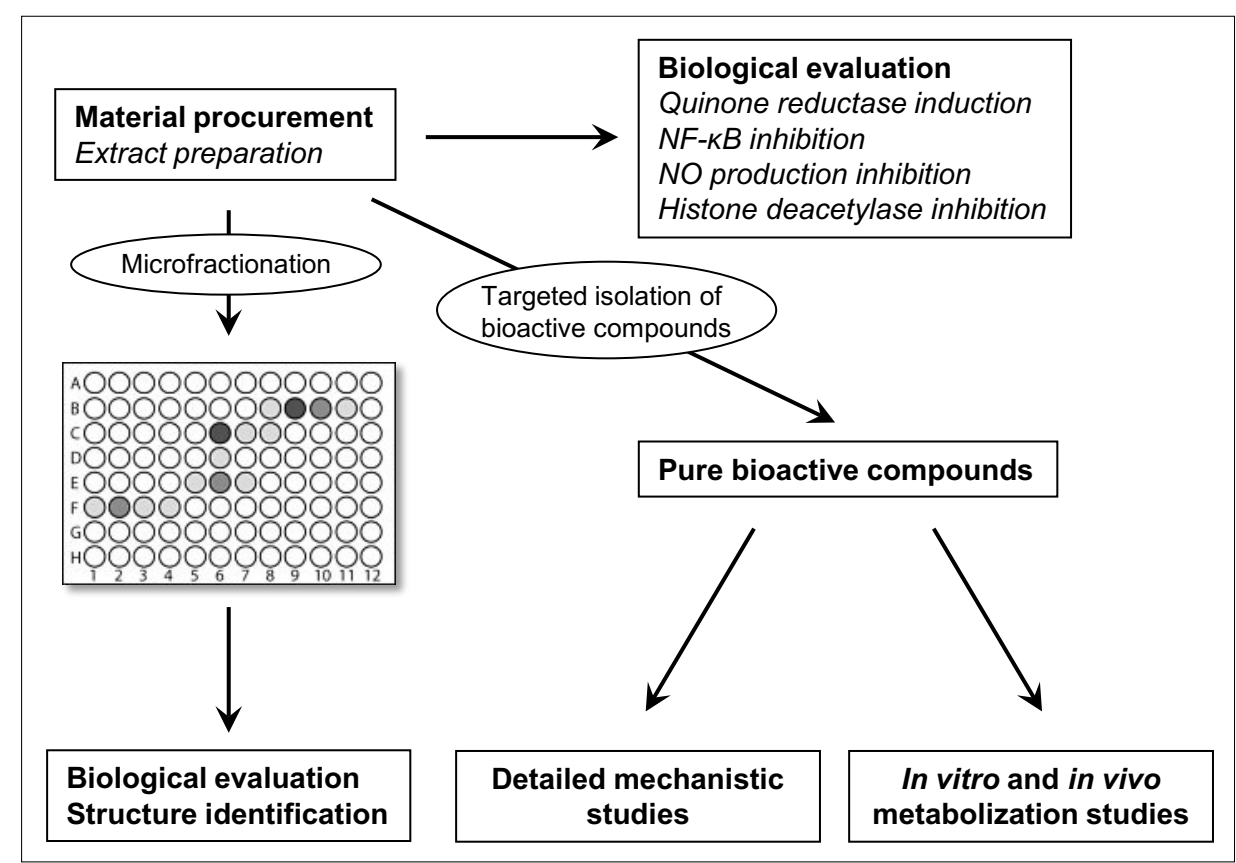

Fig. 1. Cancer chemoprevention project organization. 


\subsection{Anti-initiation Activity}

Carcinogenesis is a complex multistage process, yet the entire course can be initiated by a single event wherein a cellular macromolecule is damaged by an endogenous or exogenous agent. Strategies for protecting cells from these initiating events include the activation of phase II enzymes, such as $\mathrm{QR}$, that can deactivate radicals and electrophiles known to intercede in normal cellular processes. QR elevation has been shown to correlate with induction of other protective phase II enzymes and provides a reasonable biomarker for the potential chemoprotective effect of test agents against cancer initiation. ${ }^{[10]}$ The murine hepatoma cells Hepa 1c1c7 are used to determine QR activity by measuring the NADPH dependent menadio-mediated reduction of 3-(4,5-dimethylthiazo-2-yl)-2,5-diphenyltetrazolium bromide (MTT). ${ }^{[1]}$

\subsection{Anti-promotion Activity}

$\mathrm{NF}-\mathrm{\kappa B}$ is a ubiquitous transcription factor that is activated in a variety of cellular survival settings. ${ }^{[12]} \mathrm{NF}-\mathrm{\kappa B}$ is maintained in the cytoplasm in an inactivated or resting state by the chaperone molecule,

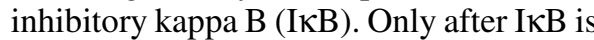
phosphorylated by inhibitor kappa kinase (IкK), is NF-KB released and free to translocate to the nucleus where it can perform its function. Upon activation, NF- $\mathrm{KB}$ has been shown to transcriptionally upregulate many genes, most of which encode molecules involved in cellular survival pathways. ${ }^{[13]}$ Many of the signaling pathways with which these molecules interface are directly involved in subverting apoptosis. The principle of the assay relies on the ability of TNF $\alpha$ to turn on the promoter $\mathrm{NF}-\mathrm{\kappa B}$, artificially constructed upstream of the luciferase gene. Once the promoter is triggered, it leads to transcription and eventually translation of the luciferase protein. The inhibition of NF- $\kappa B$ by the natural products, as indicated by the luciferase activity, can be easily measured using a luminometer. ${ }^{[7]}$

$\mathrm{NO}$, a product of nitric oxide synthase, mediates diverse physiological processes as a signaling molecule. However, continuous and excessive production of $\mathrm{NO}$ by inducible nitric oxide synthase (iNOS) causes pathophysiological problems such as chronic inflammatory diseases and cancer development. The ability of the natural products to inhibit the production of $\mathrm{NO}$ by iNOS is determined in lipopolysaccharidestimulated RAW 264.7 mouse macrophage cells. ${ }^{[14]}$

\subsection{Anti-progression Activity}

HDAC inhibitors represent a novel class of therapeutic agents with antitumor activity, but epigenetic therapy may play a significant role in cancer chemopreven- tion. Epigenetic alterations are often involved in the earliest stages of tumor progression, and usually precede neoplastic transformation. ${ }^{[8]}$ Inhibition of total HDAC activity, as well as specific HDAC activity is evaluated on HeLa cells nuclear extract or in cells incubated with the sample to be tested, by measuring a fluorophore generated by the developer when sensitized by the deacetylation of the substrate. ${ }^{[15]}$

\section{Neglected Diseases}

The World Health Organization (WHO) estimates that one sixth of the world's population suffers from one or more neglected tropical diseases. ${ }^{[16]}$ The persistence of these diseases, mainly found in the poorest regions of the world, is a consequence of the precarious life conditions of the population, the social disorganization and the lack of an effective political action and educational programs. The neglected parasitic diseases are among some of the most common infections and occur primarily in rural areas and in poor urban settings of low-income countries in sub-Saharan Africa, Asia, and Latin America, causing a marked socio-economic burden. ${ }^{[17]}$

We are currently working on two projects involving neglected diseases. The first one, in collaboration with J.-L. Wolfender is looking at innovative approaches to fight Chagas disease and leishmaniasis. The second one consists in finding the active principle from a plant decoction used to treat malaria in Africa.

\subsection{Chagas Disease and Leishmaniasis}

Chagas disease, also called American trypanosomiasis, continues to place a heavy burden on millions of people in South America. Caused by the protozoan Trypanosoma cruzi, it affects 16 to 18 million people. ${ }^{[18]}$ Due to the lack of a vaccine, patient therapy relies on chemotherapy with benznidazole and nifurtimox, but the high toxicity and low efficacy of these drugs foster the search for new drug leads. ${ }^{[19]}$ Despite few advances in drug development on antitrypanosomal chemotherapy, no drug emerged as an effective candidate to clinical trials for Chagas disease in the last 30 years. Thus, there is a great need for the development of new less toxic and more effective anti-T. cruzi agents. ${ }^{[19]}$

The WHO estimates that about 350 million of people around the world are at risk of infection with the protozoan leishmania transmited by the sandfly, and more than 2 million of new cases of leishmaniasis occur annually. Four countries (Brazil, India, Bangladesh, and Sudan) alone present 90\% of cases of visceral leishmaniasis, the most severe form of the disease. Despite the progress made in the understanding of the immunopathology of leishmaniasis, the drugs available for the treatment of this illness still present today many adverse effects due to their high toxicity, and can lead to drug resistance. ${ }^{[20]}$ The drugs prescribed for the treatment of leishmaniasis are mostly pentavalent antimonial derivatives, which have been used for more than 50 years. They are highly toxic, and numerous patients are refractory to them. The search for new chemotherapies is therefore needed.

With funding from the European Union under Framework Seven, we belong to a consortium of 11 universities that has developed a project for exploring plant chemical biodiversity and innovative approaches to fight Chagas disease and leishmaniasis - 'ChemBioFight'. Initially, in silico screening of the existing collections of pure compounds of all participants is undertaken, aiming to discover active scaffolds. The results of this process are correlated with the existing chemotaxonomic databases and the literature which in turn will lead to the focused collection of natural sources by the corresponding participants. The extracts of the collected material are evaluated according to their biological activities by in vitro and in vivo assays while application of the metabolomics approach (profiling and fingerprinting) will facilitate sample classification, unmask dereplication and correlate extract composition to biological activity. The extracts are subjected to further processing in terms of targeted isolation and purification of active compounds with state-of-the-art technologies, which in turn allow for the accurate biological evaluation of the pure compounds. Furthermore powerful spectroscopic techniques are used for the structural elucidation of the most promising molecules, based on which the design of new semisynthetic analogues is possible.

\subsection{Malaria}

Malaria is one of the oldest parasitic diseases. It is caused by protozoan of the genus Plasmodium. According to the World Malaria Report 2010, there were 225 million cases of malaria and an estimated 781,000 deaths in 2009, mostly of children under the age of 5.[21] The problem is aggravated by the emergence of strains of $P$. falciparum resistant to antimalarial drugs, as well as resistance of the female Anopheline mosquitoes vector to insecticides. Presently, the most important weapon to fight malaria still remains chemotherapy. In this context, secondary metabolites from plants represent a valuable source of lead compounds.

In collaboration with Medicines for Malaria Venture, a non-governmental organization, the Mali-Genève Association 


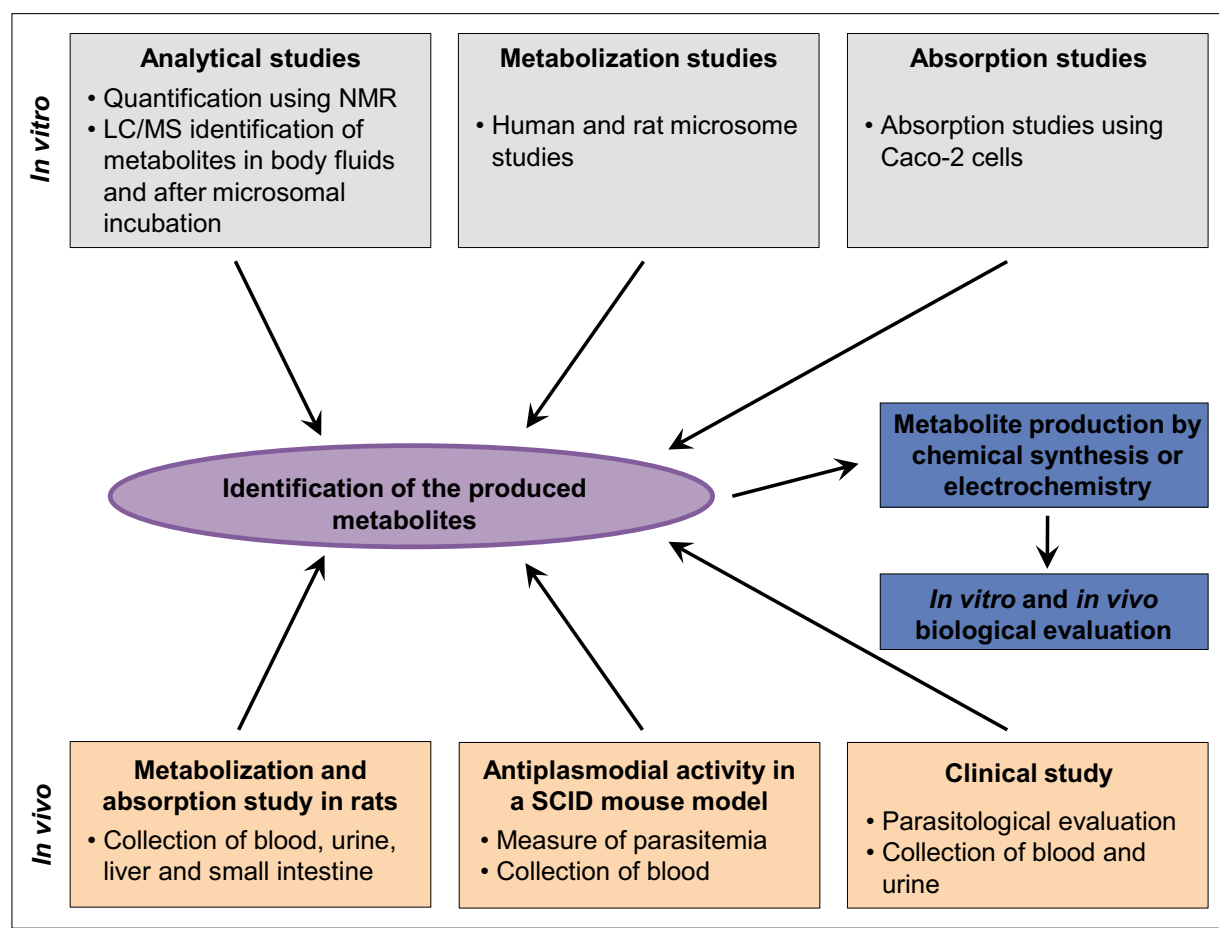

Fig. 2. Active compound identification for the treatment of malaria after proven clinical efficacy.

as well as the Malaria Research Training Center, and the Department of Traditional Medicine (DTM) of Mali, a research project has been developed based on the promising clinical results of a plant decoction used in traditional medicine to treat malaria in southern Mali. A clinical efficacy of the phytopreparation comparable to standard treatment has been established for uncomplicated malaria. Also, the in vitro efficacy of the phytopreparation against $P$. falciparum has been confirmed by several studies. Even though three in vitro active compounds have been isolated from this plant, it is insufficient to explain its clinical activity. Furthermore, the mechanisms of action are unknown.

Therefore, several challenges are encountered during the current project (Fig. 2). First, the development of specific and sensitive analytical conditions is needed to identify and quantify individual compounds and their metabolites in body fluids and after microsomal incubations. In a second step, metabolization studies of individual compounds or a combination of them in microsomes, as well as in rats are performed. The use of an in vitro absorption model using Caco- 2 cells also gives good information to design in vivo studies. Finally, parasitological evaluation and pharmacokinetic studies will be performed in a SCID mouse model, as well as in a clinical study. This will lead to the identification of metabolites, which will then be produced either by chemical synthesis or electrochemistry in order to evaluate their activity against $P$. falciparum.

\section{Targeted Screening of Botanicals in Herbal Dietary Supplements}

Herbal dietary supplements (HDS) are very trendy. The commercial success of botanical ingredients is largely due to a growing interest for products supporting a healthy lifestyle. HDS are widely marketed with various health claims. These phytopreparations are easily available to consumers through several distribution channels: OTC in pharmacies, in supermarkets, herbalist's shops or via the Internet.

In this context, problems related to undeclared, unauthorized ${ }^{[22]}$ or toxic botanicals in HDS is of growing importance. Indeed, according to the current food leg- islation, HDS are considered as foodstuff, so they generally have not gone through a rigorous drug testing process as for therapeutic phytochemicals. Furthermore, there is a need to conduct authenticity analyses to confirm the presence of the declared botanicals. Chemical methods already exist for the detection of plants but they are usually specific for a particular species or dedicated to a class of compounds.

In collaboration with the Official Food Control Authority of Geneva, a generic method was developed for the multitargeted screening of biomarkers (BM) which aims at characterizing plant species in HDS. To minimize discriminations, botanical preparations are unselectively extracted by sonication in methanol. The analytical method uses high performance liquid chromatography coupled to hybrid mass spectrometry (Q-Trap) with an electrospray ionization source. The MS acquisition mode is based upon an Information Dependent Acquisition (IDA) approach generating MS/MS spectra which can be compared with an in-house built library. The IDA mode combines a survey acquisition mode to select candidates of interest according to predefined criteria with a subsequent MS/MS full scan as the data dependent experiment (Fig. 3).

\subsection{Methodology}

The first step consists in identifying a (or several) specific biomarker(s) in the literature for the plant of interest. This marker, if commercially available and easily amendable to LC-MS, can be characterized by its chromatographic retention time $\left(t_{R}\right)$, two specific mass transitions and their corresponding ratio, as well as three Enhanced Product Ion (EPI) scans that are generated at three different collision energies. In this mode, the selection of the precursor ion is realized in the first quad-

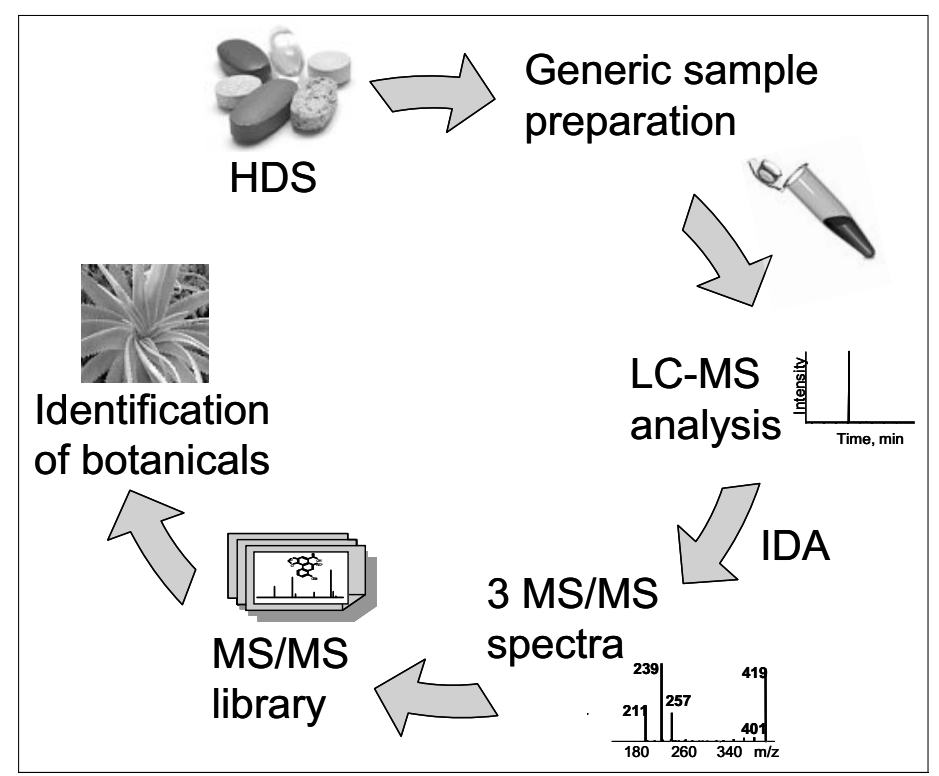

Fig. 3. Flow chart analysis of herbal dietary supplements. 


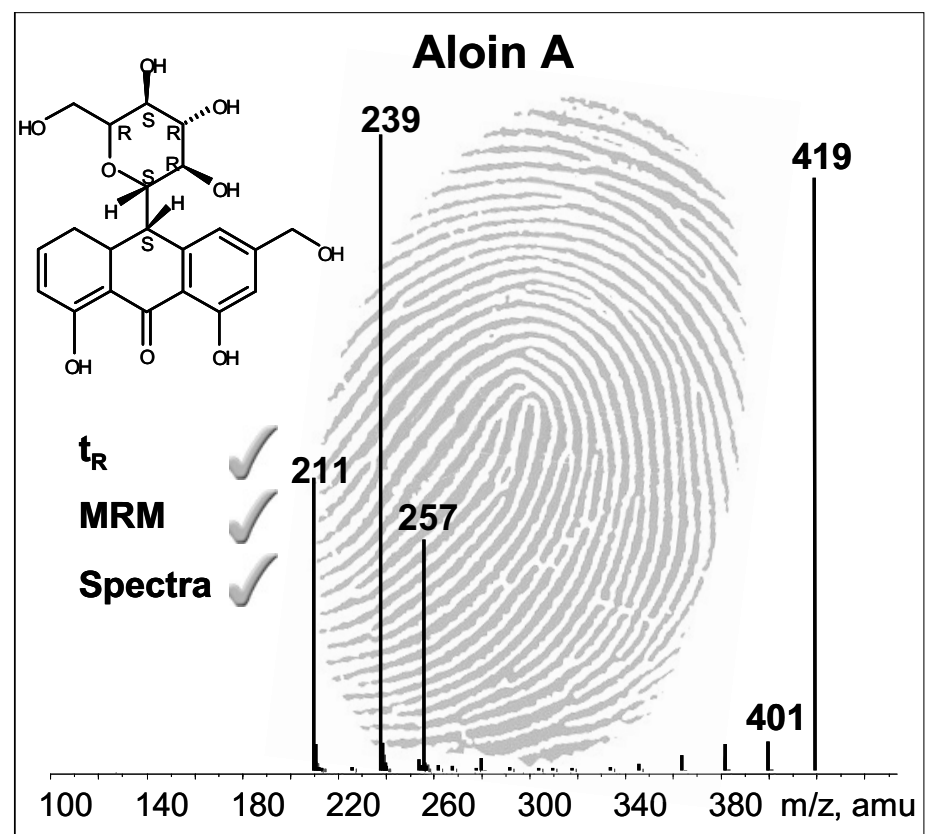

rupole $(\mathrm{Q} 1)$ and fragments are trapped in the third quadrupole (Q3) operating as a linear ion trap. This approach allows to unambiguously identify the selected biomarkers by comparing the MS/MS spectra (fingerprints) obtained from real samples with those acquired with the reference standards (Fig. 4). To fulfil our selection criteria in terms of sensitivity, the limit of detection must be below $500 \mathrm{ng} / \mathrm{ml}$.

Typically, during a screening analysis, each BM is targeted by its most sensitive mass transition (Multiple Reaction Monitoring-MRM) and if its signal exceeds 1000 counts per second, MS/MS spectra are generated at low, medium and high collision energies. During the data treatment, the three MS/MS spectra of each individual BM are compared with the library. The identification of the specific biomarkers indicates the presence of the corresponding plant in the HDS. If quantitative information is required, a second analysis is conducted in the conventional MRM mode by monitoring both transitions.
Fig. 4. Identification of the biomarker aloin A by LC-MS/MS.

\subsection{Applications}

Among the screened botanicals, unauthorized plants with therapeutic activities are frequently encountered in HDS. For instance, in Switzerland, Ginkgo biloba L. can only be used in phytopreparations registered by Swissmedic (Swiss Agency for Therapeutic Products) because of its bioactive ginkgolides and bilobalides ingredients.

Some plants are incorporated in the generic method because they have one or several compounds of interest which are only allowed in a limited amount. Typically, Aloe vera L. can only be included in HDS if the concentration of aloin is lower than the therapeutic threshold (less than $0.1 \mathrm{mg} / \mathrm{L}$ or $\mathrm{mg} / \mathrm{kg}$ )

Furthermore, the method is able to detect several toxins such as the neurotoxin anisatine. This compound enables to screen for potential confusions between various species of star anise, especially between I. verum Hook. f. and I. anisatum L.; the intake of the latter can lead to severe convulsions.

Alternatively, the present method may be used to confirm the phytochemical composition of the preparations.
Received: February 2, 2012

[1] M. B. Sporn, N. Suh, Carcinogenesis 2000, 21, 525.

[2] M. B. Sporn, N. M. Dunlop, D. L. Newton, J. M. Smith, Fed. Proc. 1976, 35, 1332.

[3] L. W. Wattenberg, Cancer Res. 1985, 45, 1.

[4] G. J. Kelloff, C. C. Sigman, P. Greenwald, Eur. J. Cancer 1999, 35, 1755.

[5] G. J. Kelloff, J. A. Crowell, E. T. Hawk, V. E. Steele, R. A. Lubet, C. W. Boone, J. M. Covey, L. A. Doody, G. S. Omenn, P. Greenwald, W. K. Hong, D. R. Parkinson, D. Bagheri, G. T. Baxter, M. Blunden, M. K. Doeltz, K. M. Eisenhauer, K. Johnson, G. G. Knapp, D. G Longfellow, W. F. Malone, S. G. Nayfield, H. E. Seifried, L. M. Swall, C. C. Sigman, J. Cell Biochem. Suppl. 1996, $26,54$.

[6] M. Cuendet, C. P. Oteham, R. C. Moon, J. M. Pezzuto, J. Nat. Prod. 2006, 69, 460.

[7] S. S. Kang, M. Cuendet, D. C. Endringer, V. L. Croy, J. M. Pezzuto, M. A. Lipton, Bioorg. Med. Chem. 2009, 17, 1044.

[8] M. Toyota, J. P. Issa, Semin. Oncol. 2005, 32, 521.

[9] J. L. Wolfender, P. J. Eugster, N. Bohni, M Cuendet, Chimia 2011, 65, 400.

[10] P. Talalay, H. J. Prochaska, S. R. Spencer, Princess Takamatsu Symp. 1990, 21, 177.

[11] H. J. Prochaska, A. B. Santamaria, P. Talalay, Proc. Natl Acad. Sci. USA 1992, 89, 2394.

[12] H. L. Pahl, Oncogene 1999, 18, 6853.

[13] S. A. Schwartz, A. Hernandez, B. Mark Evers, Surg. Oncol. 1999, 8, 143.

[14] S. Cheenpracha, E. J. Park, W. Y. Yoshida, C. Barit, M. Wall, J. M. Pezzuto, L. C. Chang, Bioorg. Med. Chem. 2010, 18, 6598.

[15] K. Hoffmann, G. Brosch, P. Loidl, M. Jung, Nucleic Acids Res. 1999, 27, 2057.

[16] L. Savioli, D. Engels, D. Daumerie, J. Jannin, J. Alvar, K. Asiedu, M. Gastellu-Etchegorry, P. Simarro, S. P. Mariotti, PLoS Med. 2006, 3 , e283.

[17] P. J. Hotez, D. H. Molyneux, A. Fenwick, J. Kumaresan, S. E. Sachs, J. D. Sachs, L. Savioli, N. Engl. J. Med. 2007, 357, 1018.

[18] C. J. Schofield, J. Jannin, R. Salvatella, Trends Parasitol. 2006, 22, 583.

[19] D. R. Moreira, A. C. Leite, R. R. dos Santos, M B. P. Soares, Curr. Drug Targets 2009, 10, 212

[20] A. Bryceson, Trop. Med. Int. Health 2001, 6, 928

[21] World Malaria Report. 2010, World Health Organization.

[22] Swiss guidance on the categorization of botanicals intended for use as therapeutic preparations or food supplements. List of botanicals, version 1.3, Federal Office of Public Health, Switzerland, 2010. 